\title{
Editorial
}

\section{Controlling Chaos and Bifurcations in Discrete-Time Population Models}

\author{
Qamar Din, ${ }^{1}$ A. A. Elsadany, ${ }^{2}$ and Hammad Khali1 ${ }^{3}$ \\ ${ }^{1}$ Department of Mathematics, The University of Poonch, Rawalakot, Pakistan \\ ${ }^{2}$ Basic Science Department, Faculty of Computers and Informatics, Suez Canal University, New Campus, Ismailia 41522, Egypt \\ ${ }^{3}$ Department of Mathematics, University of Education, Attock Campus, Lahore, Pakistan \\ Correspondence should be addressed to Qamar Din; qamar.sms@gmail.com
}

Received 23 August 2017; Accepted 23 August 2017; Published 25 October 2017

Copyright (C) 2017 Qamar Din et al. This is an open access article distributed under the Creative Commons Attribution License, which permits unrestricted use, distribution, and reproduction in any medium, provided the original work is properly cited.

The theory of discrete dynamical systems has many applications in almost all branches of the applied sciences. We can understand the nonlinear phenomena and complexity by studying the qualitative behavior of discrete dynamical systems. To a certain extent, the growing interest in difference equations may be also attributed to their simplicity. Although only quite simple computational and graphical representation tools are necessary to study the behavior of the solutions of difference equations and their bifurcations for varying specific parameters in suitable intervals, it is possible to appreciate the complicated and surprisingly diverse dynamics of difference equations. Discrete-time models can be used to study the population dynamics in which time is taken from one generation to the next. These discrete-time population models are suitable for nonoverlapping generations and are appropriate to describe the nonlinear dynamics and their chaotic behavior. Controlling chaos and bifurcations refers to the task of designing a controller to modify the chaotic behaviors and bifurcation properties of a given nonlinear system and thereby achieving some desirable dynamical behaviors.

In this editorial, we present published papers which analyze various aspects and applications related to this special issue.

The research article entitled "Nonstationary and Chaotic Dynamics in Age-Structured Population Models" by A. Wikan and $\varnothing$. Kristensen deals with the dynamics of nonlinear discrete age-structured population models. The authors focus on bifurcations, as well as nonstationary and chaotic dynamics. Moreover, different mechanisms which may lead to periodic phenomena are discussed. Some new results are also presented, in particular from models where both fecundity and survival terms contain nonlinear elements.

In "Complexity and Application of Tobacco Manufacturer Pricing Game considering Market Segments," S. Guo et al. consider a strategy using two methods of market segmentation through game theory based on the collected data of several typical cities in Shandong province. Three oligarchs are discussed to analyze the complexity of the market in tobacco system. The effect of different factors on the equilibrium price of Nash equilibrium is discussed. Furthermore, the variation of parameters drives the system to change to uncertain status, even to bifurcation or chaos. The authors also discuss the model under normal circumstances, when the three parties jointly take chaos control measures, and its impact on the system. Finally, the chaos control of economic significance is discussed. These results indicate that only reasonable pricing strategy is a critical point to achieve maximized profit and maintain orderly operation at the same time, which is a constructive suggestion for tobacco companies for their operational strategy.

The paper entitled "Bifurcation Analysis and Chaos Control in a Discrete-Time Predator-Prey System of Leslie Type with Simplified Holling Type IV Functional Response" by S. M. S. Rana and U. Kulsum deals with the dynamical behavior of a discrete-time predator-prey system of Leslie 
type with simplified Holling type IV functional response. It is mathematically proved that the system undergoes NeimarkSacker at its positive steady-state. Numerical simulations are presented not only to validate analytical results but also to show chaotic behaviors which include bifurcations, phase portraits, periods $2,4,6,8,10$, and 20 orbits, invariant closed cycle, and attracting chaotic sets. Furthermore, numerically maximum Lyapunov exponents and fractal dimension are computed to justify the chaotic behaviors of the system. Furthermore, a strategy of feedback control is applied to stabilize chaos existing in the system.

By the paper entitled "Chaotic Dynamics and Control of Discrete Ratio-Dependent Predator-Prey System,” S. M. S. Rana examined the complexity of a discrete-time predatorprey system with ratio-dependent functional response. The parametric conditions for existence of fixed points and their stability are demonstrated. Moreover, the parametric conditions for existence and direction of Neimark-Sacker are investigated. Numerical simulations are presented not only to justify theoretical results but also to exhibit new complex behaviors which include phase portraits, orbits of periods 9 , 19 , and 26, invariant closed circle, and attracting chaotic sets. Furthermore, maximum Lyapunov exponents and fractal dimension are computed to confirm the chaotic dynamics of the system. Finally, a state feedback control method is applied to control chaos which exists in the system.

In "Analysis of Nonlinear Duopoly Games with Product Differentiation: Stability, Global Dynamics, and Control," S. S. Askar and A. Al-khedhairi consider a cubic utility function that is derived from a constant elasticity of substitution production function (CES). This cubic function is more desirable than the quadratic one besides its amenability to efficiency analysis. Based on that utility, a two-dimensional Cournot duopoly game with horizontal product differentiation is modeled using a discrete-time scale. Two different types of games are studied. In the first game, firms are updating their output production using the traditional bounded rationality approach. In the second game, firms adopt Puu's mechanism to update their productions. Puu's mechanism does not require any information about the profit function; instead, it needs both firms to know their production and their profits in the past time periods. In both scenarios, an explicit form for the Nash equilibrium point is obtained under certain conditions. The stability analysis of Nash point is considered. Furthermore, some numerical simulations are carried out to confirm the chaotic behavior of Nash equilibrium point. This analysis includes bifurcation, attractor, maximum Lyapunov exponent, and sensitivity to initial conditions.

The paper entitled "Robust Active MPC Synchronization for Two Discrete-Time Chaotic Systems with Bounded Disturbance" by L. Zhang deals with a synchronization scheme for two discrete-time chaotic systems with bounded disturbance. By using active control method and imposing some restriction on the error state, the computation of controller's feedback matrix is converted to the min-max optimization problem. The theoretical results are derived with the help of predictive model predictive paradigm and linear matrix inequality technique. Numerical examples are provided to show the effectiveness of the designed control method.

In "An Analysis of Discrete Stage-Structured Prey and Prey-Predator Population Models," A. Wikan studied discrete stage-structured prey and prey-predator models. It is proved that the models under consideration are permanent (i.e., the population will neither go extinct nor exhibit explosive oscillations) and, moreover, that the transfer from stability to nonstationary behavior always goes through a supercritical Neimark-Sacker bifurcation. The prey model covers species that possess a wide range of different life histories. Predation pressure may both stabilize and destabilize the prey dynamics but the strength of impact is closely related to life history. Indeed, if the prey possesses a precocious semelparous life history and exhibits chaotic oscillations, it is shown that increased predation may stabilize the dynamics and also, in case of large predation pressure, transfer the population to another chaotic regime.

The research article entitled "Neimark-Sacker Bifurcation and Chaos Control in a Fractional-Order Plant-Herbivore Model" by Q. Din et al. deals with the dynamics of a discretetime 3-dimensional plant-herbivore model. Moreover, the existence and uniqueness of positive equilibrium and parametric conditions for local asymptotic stability of positive equilibrium point of the model are investigated. Moreover, it is also proved that the system undergoes Neimark-Sacker bifurcation for positive equilibrium with the help of an explicit criterion for Neimark-Sacker bifurcation. The chaos control in the model is discussed through implementation of two feedback control strategies, that is, pole-placement technique and hybrid control methodology.

In "Complex Dynamics and Chaos Control on a kind of Bertrand Duopoly Game Model Considering R\&D Activities," H. Tu et al. study the dynamics and development of two-stage input competition game model in the Bertrand duopoly oligopoly market with spillover effects on cost reduction. The stability of the Nash equilibrium point and local stable conditions and stability region of the Nash equilibrium point by the bifurcation theory are investigated. The complex dynamic behaviors of the system are shown by numerical simulations. It is demonstrated that chaos occurs for a range of managerial policies, and the associated unpredictability is solely due to the dynamics of the interaction. It is investigated that the straight line stabilization method is the appropriate management measure to control the chaos.

The research paper entitled "Decomposition Technique and a Family of Efficient Schemes for Nonlinear Equations" by F. A. Shah et al. deals with a new family of iterative methods for solving nonlinear equations which is developed by using a new decomposition technique. The convergence of the new methods is proved. For the implementation and performance of the new methods, some examples are solved and the results are compared with some existing methods.

The guest editors of this special issue hope that problems discussed and investigated in the papers by the authors of this issue can inspire and motivate researchers in these fields to discover new, innovative, and novel applications in all areas of pure and applied mathematics. 


\section{Acknowledgments}

We would like to express our great gratitude to all of the authors for their contributions and the reviewers for their serious evaluation of the papers submitted by valuable comments and timely feedback.

Qamar Din

A. A. Elsadany

Hammad Khalil 


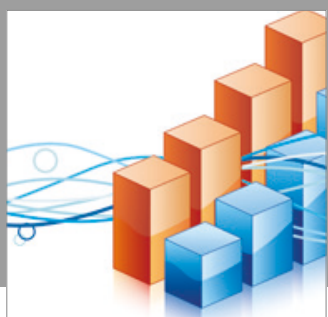

Advances in

Operations Research

vatersals

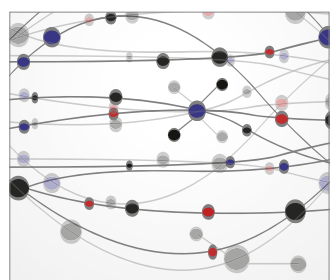

\section{The Scientific} World Journal
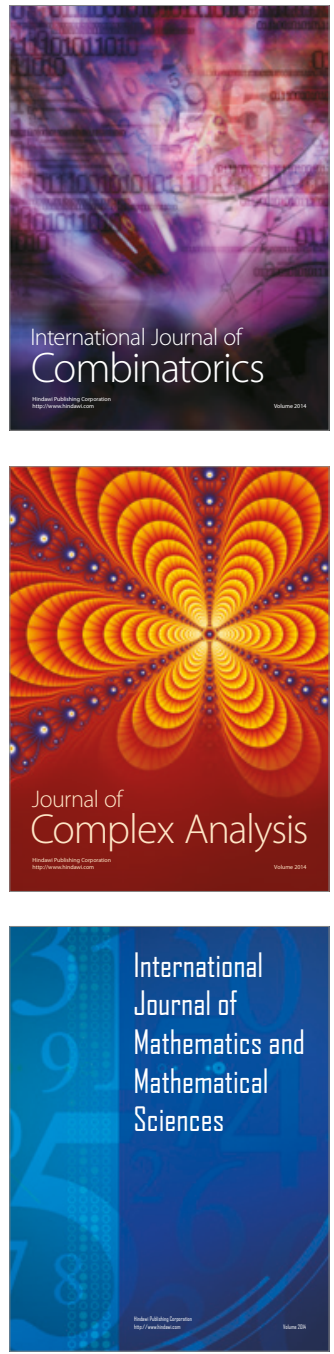
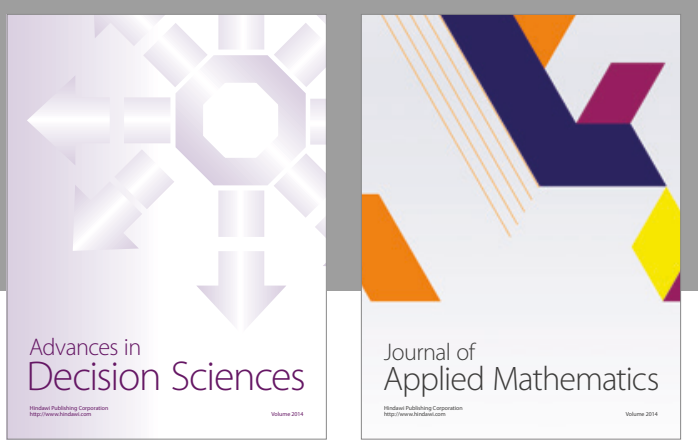

Algebra

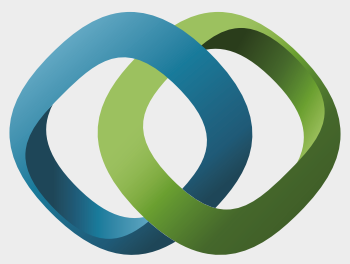

\section{Hindawi}

Submit your manuscripts at

https://www.hindawi.com
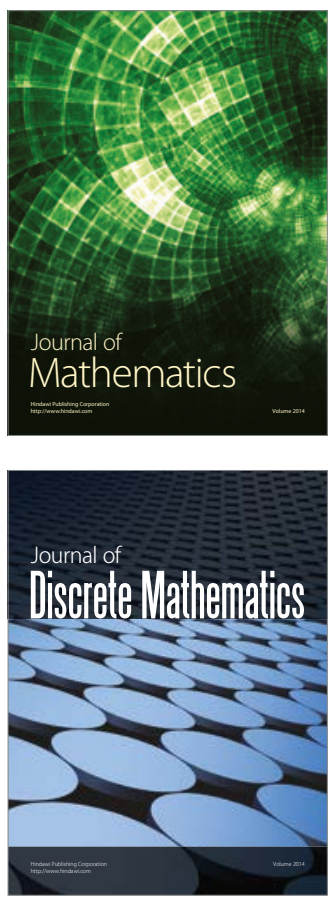

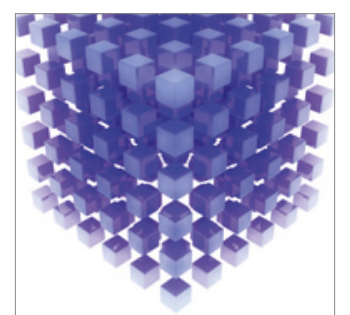

Mathematical Problems in Engineering
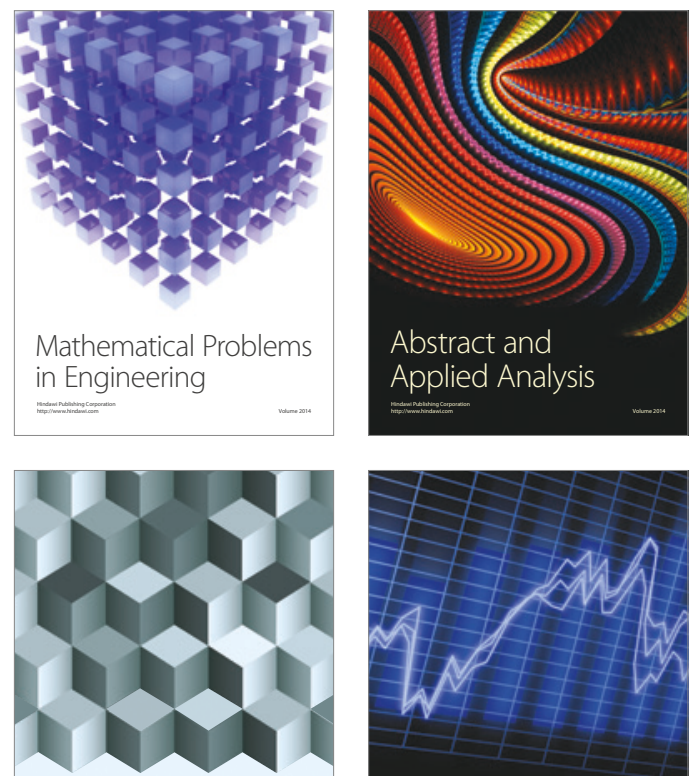

Journal of

Function Spaces

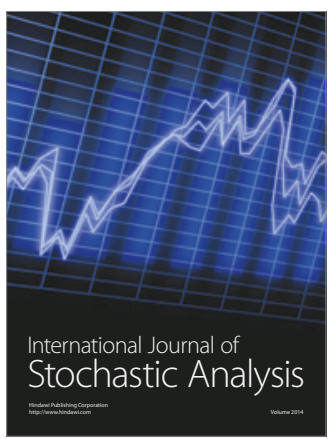

Probability and Statistics
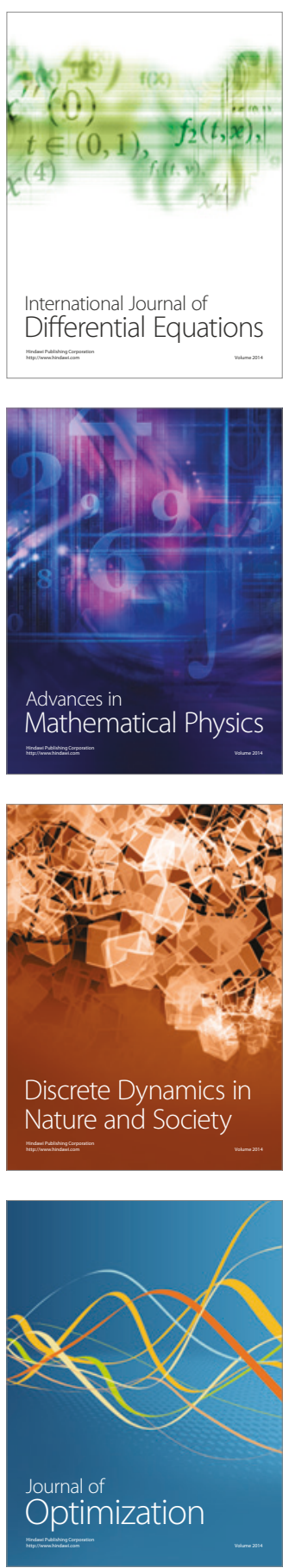